\section{Pulsating Microbubble in a Micro-vessel and Mechanical Effect on Vessel Wall: A Simulation Study}

\author{
Zahra Khodabakhshi',2๑, Nazanin Hosseinkhah³, Hossein \\ Ghadiri $4,5 * \bullet$
}

\begin{abstract}
Background: Microbubbles are widely used in diagnostic ultrasound applications as contrast agents. Recently, many studies have shown that microbubbles have good potential for the use in therapeutic applications such as drug and gene delivery and opening of blood- brain barrier locally and transiently. When microbubbles are located inside an elastic microvessel and activated by ultrasound, they oscillate and induce mechanical stresses on the vessel wall. However, the mechanical stresses have beneficial therapeutic effects, they may induce vessel damage if they are too high. Microstreaming-induced shear stress is one of the most important wall stresses.

Objective: The overall aim of this study is to simulate the interaction between confined bubble inside an elastic microvessel and ultrasound field and investigate the effective parameters on microstreaming-induced shear stress.

Material and Methods: In this Simulation study, we conducted a 2D finite element simulation to study confined microbubble dynamics, also we investigated both acoustical and bubble material parameters on microbubble oscillation and wall stress.

Results: Based on our results, for acoustic parameters in the range of therapeutic applications, the maximum shear stress was lower than $4 \mathrm{kPa}$. Shear stress was approximately independent from shell viscosity whereas it decreased by increasing the shell stiffness. Moreover, shear stress showed an increasing trend with acoustic pressure.
\end{abstract}

Conclusion: Beside the acoustical parameters, bubble properties have important effects on bubble behavior so that the softer and larger bubbles are more appropriate for therapeutic application as they can decrease the required frequency and acoustic pressure while inducing the same biological effects.

Citation: Khodabakhshi Z, Hosseinkhah N, Ghadiri H. Pulsating Microbubble in a Micro-vessel and Mechanical Effect on Vessel Wall: A Simulation Study. J Biomed Phys Eng. 2021;11(5):629-640. doi: 10.31661/jbpe.v0i0.1131.

\section{Keywords}

Ultrasound; Microbubbles; Shear Stress; Therapeutics; Blood-Brain-Barrier

\section{Introduction}

$\mathrm{E}$ ncapsulated microbubbles were initially used as contrast agents in diagnostic ultrasound imaging because they had good backscatter properties and higher echogenicity than the background tissue due to their high compressibility. Commercially available MBs consist of a gas core, which has low solubility in blood, within a shell composed of lipid, protein, albumin or polymer. The presence of shell minimizing the surface tension between gas and liquid interface and
${ }^{1} \mathrm{MSc}$, Department of Medical Physics and Biomedical Engineering, Tehran University of Medical Science, Teh-

ran, Iran

${ }^{2} \mathrm{MSc}$, Research Center

for Molecular and Cel-

lular Imaging ( $\mathrm{RCMCl}$ ),

Tehran, Iran

${ }^{3} \mathrm{PhD}$, Department of

Medical Biophysics,

University of Toronto,

Toronto, ON, Canada

${ }^{4} \mathrm{PhD}$, Department of

Medical Physics and

Biomedical Engineer-

ing, Tehran University of

Medical Science, Teh-

ran, Iran

${ }^{5} \mathrm{PhD}$, Research Center

for Molecular and Cel-

lular Imaging (RCMCl),

Tehran, Iran

*Corresponding author: Hossein Ghadiri

Department of Medical

Physics and Biomedi-

cal Engineering, Tehran

University of Medical

Science, Tehran, Iran

E-mail: h-ghadiri@tums. ac.ir

Received: 29 January 2019 Accepted: 18 February 2019 
low solubility of gas, stabilizes the MBs and prolongs their lifetime. Ultrasound contrast agents are typically $1-10 \mu \mathrm{m}$ in diameter [1]. Recently, it has been shown that microbubbles have potential for the use in therapeutic applications [2]. Microbubble oscillations induced by ultrasound excitation can result in sonoporation and increasing the endothelial cells permeability $[3,4]$, drug and gene delivery $[5,6]$ and local and transient opening of blood brain barrier for targeted drug delivery [7].

The expansion and contraction of encapsulated microbubbles in an ultrasound field will induce microstreaming in the surrounding fluid [8]. If cells are present near oscillating microbubble, microstreaming-induced shear stress may cause biological effects on neighboring cells [9]. For instance, it will induce sonoporation on endothelial cells [4]. The sonoporation might increase the number of vesicles if the microbubble oscillates stably near the cell at low acoustic pressures [10] and it can also lead to severe damage and even cell death [11]. Therefore, it is of crucial importance to have a good knowledge about the interaction between vessel walls and the ultrasound contrast agent microbubbles exposed to the ultrasound pulse.

Beside the experimental studies, several numerical simulations have been conducted to shed light on the interaction between microbubble and ultrasound and the resulting effects on the vessel wall. For example, Sassaroli et al. proposed a model for a microbubble inside a vessel and investigated the effects of vessel radius on microbubble resonance frequency and damping factor. However, they did not consider the deformation of microvessel and viscoelastic properties of microbubble contrast agent in their study [12]. Qin et al. developed a lumped-parameter model to study the interaction between oscillating microbubble and microvessel and investigated the effects of acoustic parameters on the mechanical stress exerted on the vessel wall [13]. In another study, Martynov et al. used a lumped- parameter membrane type model to study the confinement effect of an elastic vessel on the oscillation of microbubble [14]. Doynikov et al. theoretically investigated shear stress generated by a detached microbubble on a cell membrane which had been considered as a rigid wall [15]. Weidemair et al. used a finite volume method to simulate oscillation of microbubble inside a linear elastic microvessel. They also considered the presence of red blood cells (RBCs) near the microbubble, and their resulting effects on spatial gradient of mechanical wall stresses [16]. Hosseinkhah et al. proposed a three dimensional model for ultrasound contrast agent and investigated the effects of acoustical parameters and vessel viscoelasticity on bubble behavior and the resulting shear and circumferential stress on the vessel wall [17]. There are many other simulation studies, which used FEM, BEM, FVM or combination of these methods to model of microbubble contrast agent and investigate the effects of different parameters on its behavior [18-22].

Some of these studies did not consider viscolelastic parameters of vessel or contrast agent and only investigated the effects of some acoustical parameters on bubble dynamic. The current study aims to simulate spherical oscillation of a microbubble inside a microvessel and provide a comprehensive analysis regarding the effects of varying acoustic pressure, acoustic frequency and the shell viscoelasticity on the bubble dynamic and the resulting mechanical stress on the vessel wall.

\section{Material and Methods}

\section{FEM Simulation}

In our simulation study, we modeled spherical oscillation of a microbubble inside an elastic microvessel. The microbubble with the initial radius of $1.5 \mu \mathrm{m}$ was placed in the center of a microvessel with the radius of $3 \mu \mathrm{m}$ and thickness about $1 \mu \mathrm{m}$. The vessel length was set $200 \mu \mathrm{m}$, which was large enough to make 
the results independent of vessel length. The blood domain was considered as an incompressible, single phased Newtonian fluid. At this domain, the Navier-Stokes equation was solved because the fluid domain should obey the mass and momentum conservation laws:

$$
\begin{gathered}
\frac{\partial \rho}{\partial t}+\nabla \cdot(\rho \mathbf{v})=0 \\
\rho\left(\frac{\partial \mathbf{v}}{\partial t}+(\mathbf{v} \cdot \nabla) \mathbf{v}\right)=\nabla \cdot\left(-p \boldsymbol{I}+\mu\left(\nabla \mathbf{v}+\nabla \mathbf{v}^{T}\right)\right)
\end{gathered}
$$

In the equations $\mathbf{V}$ is the fluid velocity, $\mathrm{p}$ is the fluid pressure, $\rho$ and $\mu$ are fluid density and fluid dynamic viscosity, respectively.

On the bubble boundary, bubble oscillation should satisfy the following pressure condition:

$p_{\text {avg }}=p_{g}-\frac{2 \sigma}{R}-\frac{4 \mu \dot{R}}{R}-\frac{4 K_{s} \dot{R}}{R^{2}}-4 \chi\left(\frac{1}{R_{0}}-\frac{1}{R}\right)-p_{a c}(t)(3)$

The first term on the right hand-side of equation (3) is the gas pressure inside the bubble. The gas inside the bubble considered as an ideal gas and obeyed the polytropic gas law, $p_{g}=p_{g 0}\left(\frac{R_{0}}{R}\right)^{3 k}$, where $p_{g 0}$ is the initial gas pressure at the resting state, which is $p_{0}+\frac{2 \sigma}{R_{0}}$. $p_{0}$ is the ambient pressure in capillary and set equal to $104.6 \mathrm{kPa}$ [23]; in addition, $\sigma$ is the fluid surface tension and $\kappa$ is the gas Polytropic index, which were set $0.072 \mathrm{~N} / \mathrm{m}$ and 1.07, respectively.

The second term is pressure caused by fluid surface tension, and third term is pressure cause by fluid viscosity. Moreover, $\dot{R}$ and $R$ denote bubble wall velocity and instantaneous bubble radius. Fourth and fifth terms account for bubble shell viscuse and elastic properties according to shell model proposed by Nico de Jong for lipid-shell microbubble contrast agent [24]. In this equation, $k_{s}$ and represent shell viscosity and elasticity, respectively. The last term is the incident acoustic pressure. In this study, the acoustic pressure was considered as a continuous sinusoidal wave described by the equation $p_{a c}(\mathrm{t})=p_{a} \sin \omega t$, where $p_{a}$ is the amplitude of acoustic pressure and $\omega$ is the angular frequency of the acoustic wave. As the wavelength of acoustic pressure is much larger (in order about few $\mathrm{mm}$ ) than the microbubble radius, we can assume that the acoustic pressure is uniform around the bubble.

In our simulation, at each time step, the average pressure at the bubble surface was calculated and then $R$ was calculated for each time step by substituting this value in equation (3) $\dot{R}$. The bubble-fluid interface should obey velocity continuity condition; therefore, the bubble wall velocity was set equal to the fluid velocity.

The vessel wall was considered as a linear elastic material and coupled to the fluid in a two-way manner by a velocity boundary condition:

$$
\boldsymbol{V}=\frac{\partial \mathbf{u}_{\text {solid }}}{\partial t}
$$

In the above equation $\mathbf{u}_{\text {solid }}$ is the vessel displacement vector and $\mathbf{V}$ is the fluid velocity vector.

The pressure exerted by fluid on the vessel wall is calculated by the following equation:

$$
\mathbf{f}=\left[-p \boldsymbol{I}+\mu(\nabla \mathbf{v})+(\nabla \mathbf{v})^{T}\right] . \mathbf{n}
$$

In this equation, $\mathbf{n}$ is the unit normal vector on the fluid-solid boundary. The above equation is equal to the solid normal stress:

$$
\sigma . \mathbf{n}=\left[-p \boldsymbol{I}+\mu(\nabla \mathbf{v})+(\nabla \mathbf{v})^{T}\right] . \mathbf{n}
$$

$\sigma$ is the solid stress tensor and is calculated from the linear elastic constitutive equation:

$$
\sigma_{i j}=\frac{E}{1+v}\left(\varepsilon_{i j}+\frac{v}{1-2 v} \varepsilon_{k k} \delta_{i j}\right)
$$

Where $\delta_{i j}$ is the Kronecker delta (when $i=$ $j, \delta_{i j}=1$ and when $\left.i \neq j, \delta_{i j}=0\right) \varepsilon_{i j}$ is the strain tensor, $v$ is the Poission's ratio and set to be 0.49 based on the average value of human blood vessel [25]. $E$ is the Young's modulus of the vessel wall and its value is between 1-10 MPa for biological tissues [26]. In this study, $E$ was set to be $5 \mathrm{MPa}$. In our model, both ends 
of vessel wall were fixed and the initial value of pressure and the pressure at the both ends of microvessel were set equal to the $P_{0}$.

Nearly, 7500 triangular mesh elements were solved for this physical model. We increased the mesh density near the fluid-gas interface to have results that are more precise. As the boundaries in our computational domain are moving, we have used an arbitrary Lagrangian-Eulerian method (ALE) to handle the mesh movement. This method is an intermediate between the Lagrangian and Eulerian methods and its advantage is that there is no need for new mesh generating for every configuration. This model was time-dependent and its computational time resolution was set to be 0.01 $\mu$ sec.

This model has been solved for different acoustic properties and shell viscoelastic properties; then, the microstreaming induced shear stress on the vessel was calculated for each case according to the following equation:

$$
\tau_{r z}=\mu\left(\frac{\partial u}{\partial z}+\frac{\partial w}{\partial r}\right)
$$

In this equation, $\mathrm{r}$ and $\mathrm{z}$ are the radial and axial components of cylindrical coordinate and, $\mathrm{u}$ and $\mathrm{w}$ are corresponding fluid velocity components, respectively.

\section{Model Validation}

In order to validate our numerical model, we have compared it with the result of modified Rayleigh-Plesset equation for an unbound 1.5 $\mu \mathrm{m}$ bubble. First, we have modeled a $1.5 \mu \mathrm{m}$ bubble at the center of a $200 \mu \mathrm{m}$ sphere assumed as unlimited fluid compare to the bubble dimension. The acoustic wave exerted on the bubble has $0.25 \mathrm{MPa}$ peak negative pressure amplitude and $1 \mathrm{MHz}$ frequency. In addition, the Rayleigh-Plesset equation of this bubble has been solved by MATLAB, and then the radial oscillation of the microbubble was obtained from COMSOL model and the result of Rayleigh-Plesset equation compare to each other (Figure 1). As you can see from Figure1, the COMSOL result is in good agreement with the solution of R-P equation.

\section{Results}

Confinement effects of vessel on $\mathrm{mi}$ crobubble

In this section, we compared the results of an unbound bubble to a confined bubble in a microvessel with 50 and $5 \mathrm{MPa}$ Young's modulus. The vessel radius was set to be $3 \mu \mathrm{m}$, and initial bubble radius equals $1.5 \mu \mathrm{m}, f=1$ $M H z, P_{a c}(t)=0.25 \sin (2 \mu f t) M P a$. To highlight the damping effect of the microvessel on the bubble radial oscillation, we investigated the radial oscillation of microbubble inside a nearly rigid microvessel with $50 \mathrm{MPa}$ Young's

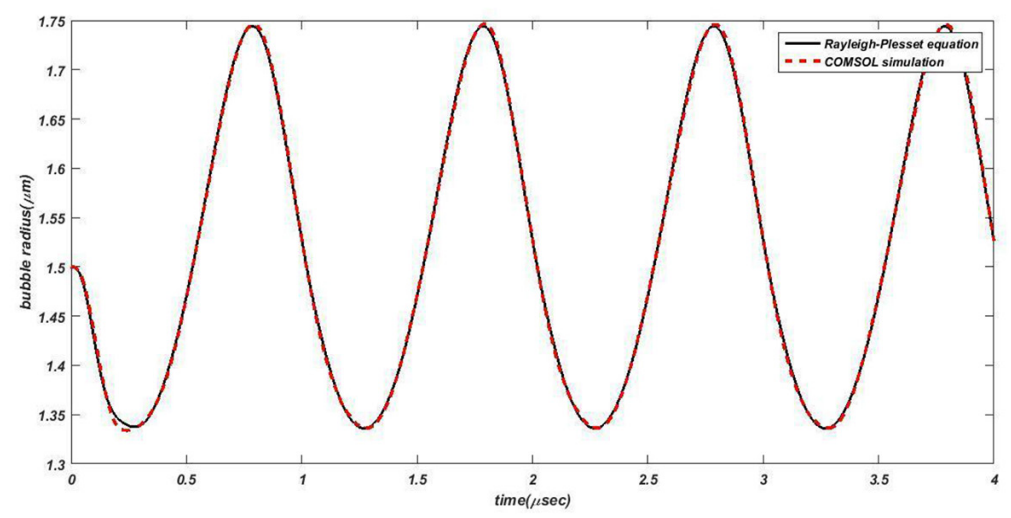

Figure 1: Comparison between R-P solution and Finite Element Method (FEM) model for $1.5 \mu \mathrm{m}$ unbound bubble. $P_{a}=0.25 \mathrm{MPa}, \mathrm{f}=1 \mathrm{MHz}$ 
modulus. As seen in Figure 2a, the maximum bubble radius decreases about $0.87 \%$ when the bubble oscillates in a rigid microvessel.

We also investigated the effect of vessel on microbubble resonance frequency. We used a range of acoustic frequency between 0.5-6 $\mathrm{MHz}$ and calculated the difference between the maximum and minimum bubble radius for a free bubble and that of a bubble inside 5 $\mathrm{MPa}$ microvessel. The resonance frequency of the free bubble is about $3.5 \mathrm{MHz}$, but the presence of $5 \mathrm{Mpa}$ vessel caused the resonance frequency of the bubble to shift toward higher frequencies, as seen in Figure $2 b$.

During bubble oscillation, the bubble pushes the fluid away and the fluid behaves like a transmitting medium and causes the vessel to start oscillating. Figure 3 shows the vessel displacement profile along the axial direction (z) at different snapshots in times. As shown in the Figure 3, the maximum vessel displacement is nearly $0.035 \mu \mathrm{m}$ and occurs exactly above the bubble $z=0, r=3 \mu m(z=0, r=3$ $\mu m)$. As time progresses, the wave propagates along the vessel and it completely dies down at $\mathrm{z}=40 \mu \mathrm{m}$.

\section{The effects of acoustic pressure}

The effect of acoustic driving pressure on bubble dynamic and microstreaming induced shear stress on vessel wall was investigated with the fixed setting of $f=1 \mathrm{MHz}, R_{0}=1.5$ $\mu m$ and $E=5 \mathrm{Mpa}$. The acoustic driving pressure varied from 0.05 to $0.45 \mathrm{MPa}$. As shown in Figure 4, by increasing the acoustic pressure, the shear stress exerted on the vessel wall

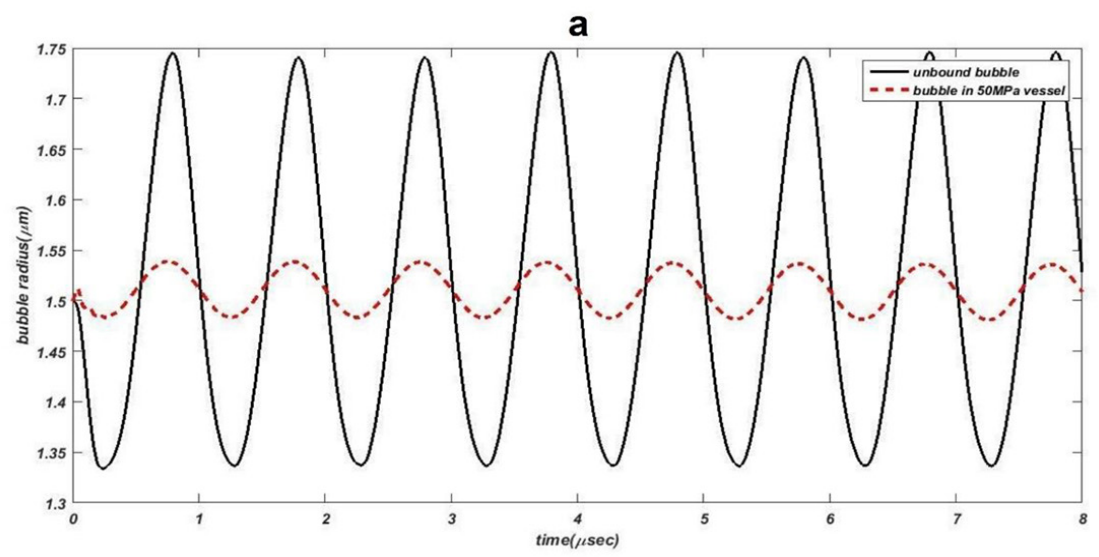

b

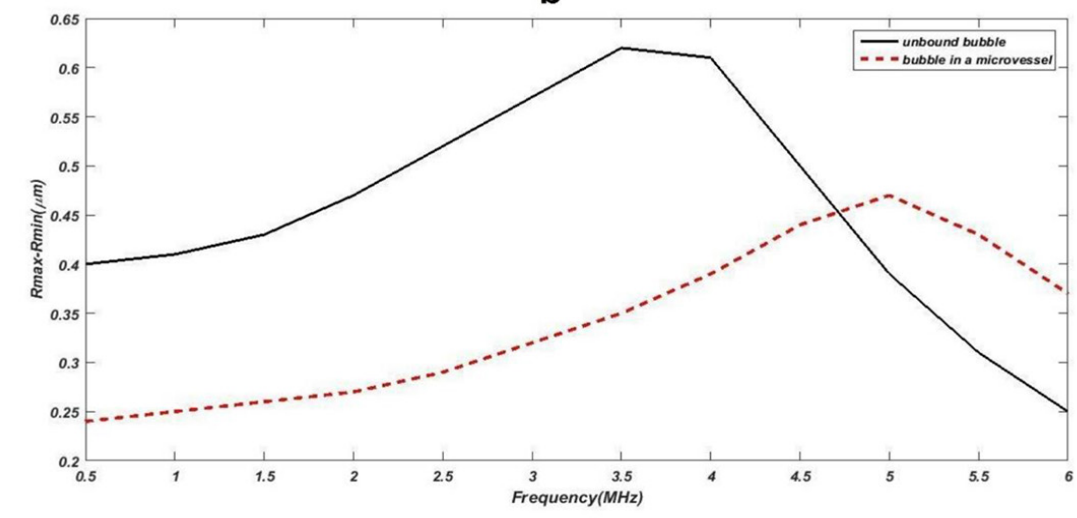

Figure 2: a) Comparison between amplitude of oscillation for unbound bubble and bubble inside an elastic microvessel. $R_{0}=1.5 \mu \mathrm{m}, E=50 \mathrm{MPa}, P_{a}=0.25 \mathrm{MPa}, f=1 \mathrm{MHz}$. b) Confinement effect of microbubble by microvessel on the resonance frequency of $1.5 \mu \mathrm{m}$ bubble 


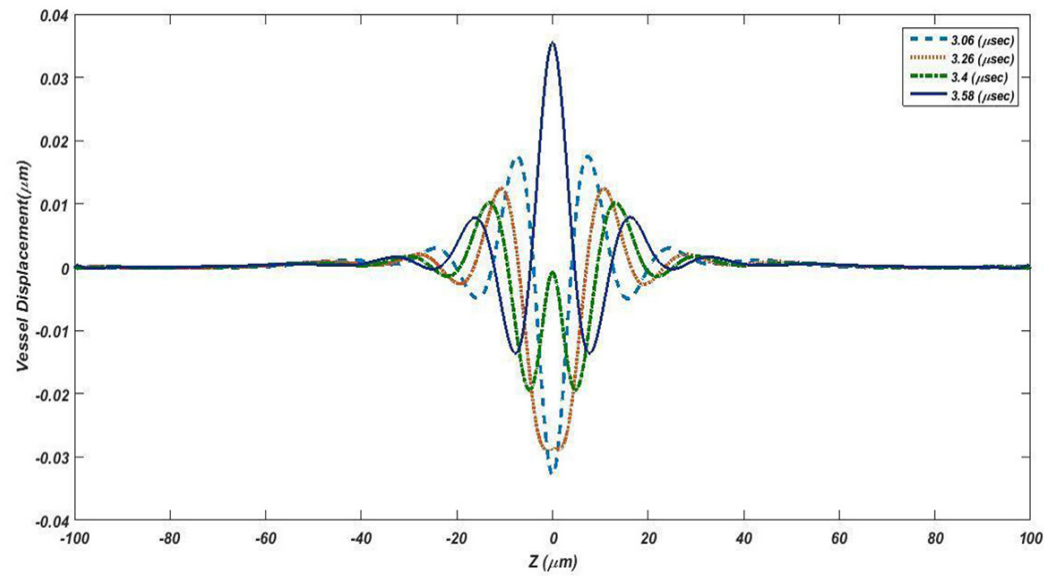

Figure 3: Spatial variation of vessel displacement along $\mathrm{z}$ axis at different times. $r_{v}=3 \mu \mathrm{m}, R_{0}=1.5$ $\mu m, E=5 \mathrm{MPa}, f=1 \mathrm{MHz}, P_{a}=0.25 \mathrm{MPa}$

increases from 0.4 to $3.5 \mathrm{kPa}$. The profile of exerted shear stress on the vessel along the axial direction at different snapshots is shown in Figure 5, Shear stress just above the bubble at $z=0$ and $r=r_{v}$ equals zero. The maximum shear stress occurres at $r=r_{v}$ and $z>0$.

In addition, the effect of different acoustic pressure was investigated at microbubble resonance frequency. As shown in Figure 6a, the exerted shear stress increases from 2.8 to 22.5 $\mathrm{kPa}$. When the acoustic frequency approaches the resonance frequency of microbubble, the microbubble will oscillate more vigorously and with the highest amplitude, thus result in the increased shear stress. In the current model, shear stress exerted on the vessel wall at bubble resonance frequency is approximately 7 times greater than that of the same bubble oscillating by $1 \mathrm{MHz}$ acoustic frequency.

The effects of acoustic frequency

Acoustic driving frequency has major impact on bubble oscillation and therefore on mechanical effects. In this section, we investigated the effects of different frequencies on the vessel wall shear stress by this setting: $p_{a}=0.2 \mathrm{MPa}$, $R_{0}=1.5 \mu \mathrm{m}$ and $R_{v}=3 \mu \mathrm{m}$. The acoustic driving frequency changed between 1-6 MHz and

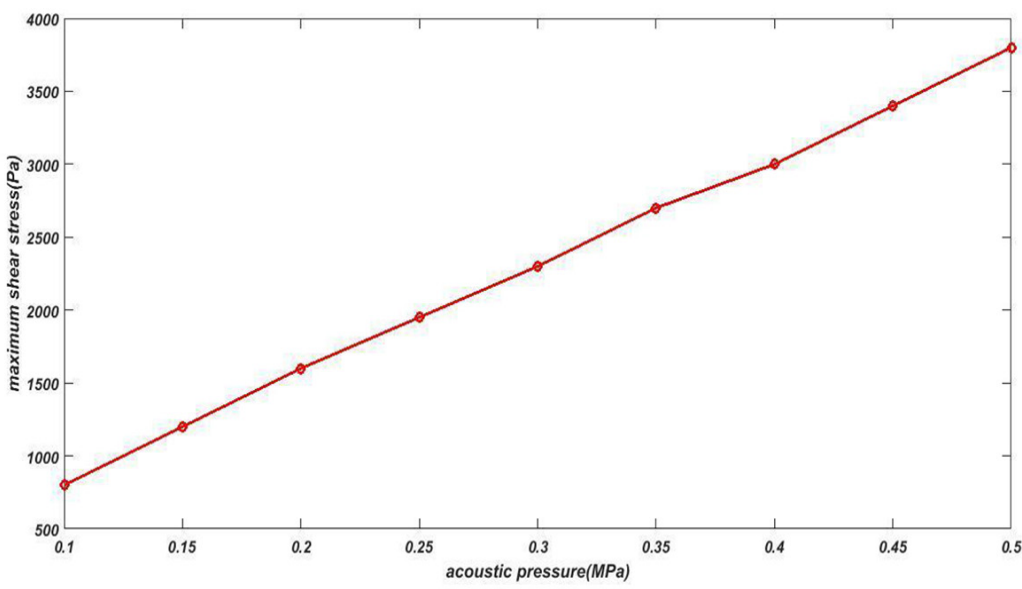

Figure 4: Maximum shear stress versus acoustic pressure amplitude 
Pulsating Microbubble in A Microvessel and its Mechanical Effects

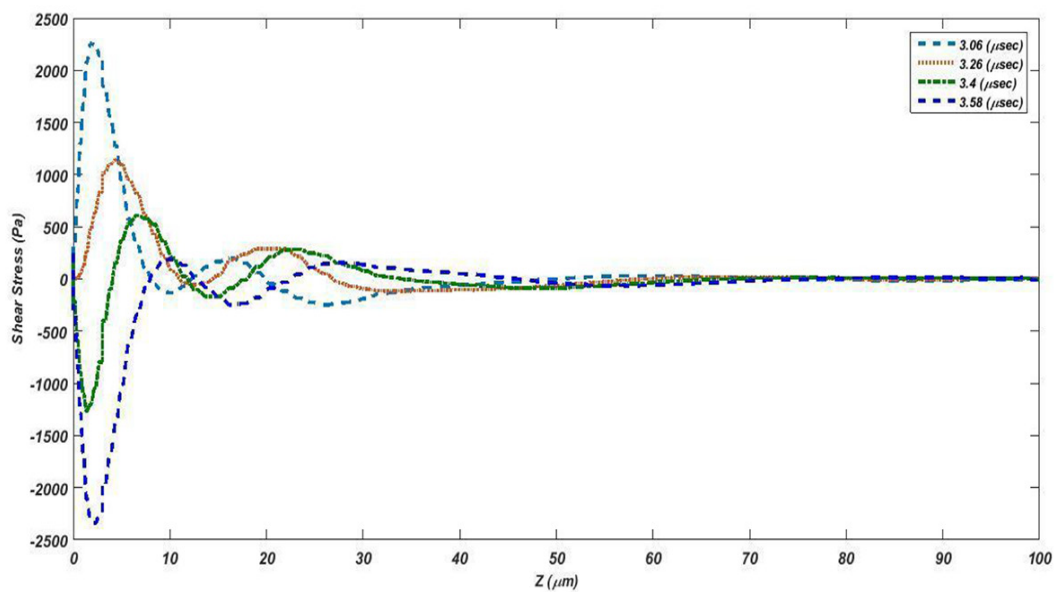

Figure 5: Shear stress profile along axial direction for different times. $P_{a}=0.3 \mathrm{MPa}, f=1 \mathrm{MHz}$

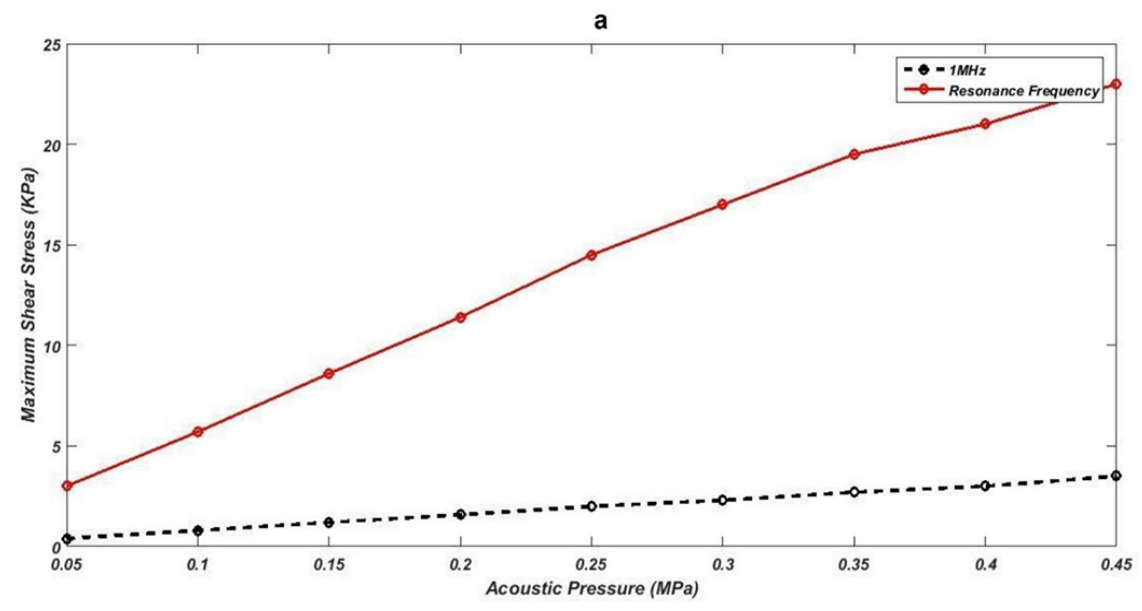

b

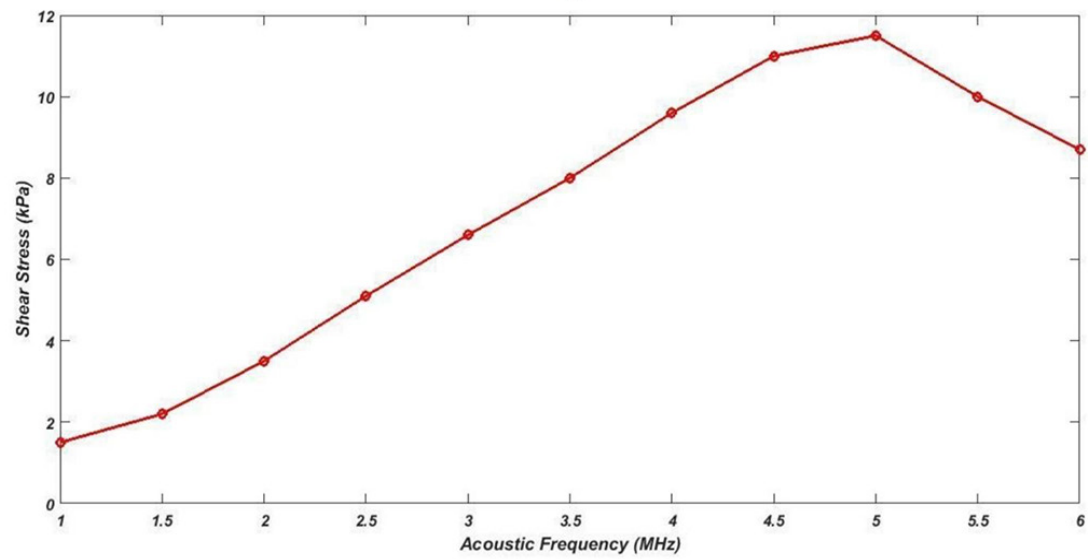

Figure 6: a) Maximum shear stress versus acoustic pressure amplitude for $f=1 \mathrm{MHz}$ and bubble resonance frequency. b) Maximum shear stress versus acoustic frequency, $P_{a}=0.2 \mathrm{MPa}$ 
the maximum shear stress exerted on the vessel wall at $\mathrm{r}=3$ and $\mathrm{z}=3 \mu \mathrm{m}$ was calculated. In Figure $6 b$, the maximum shear stress was plotted as a function of acoustic driving frequency. As it can be seen in the Figure $6 \mathrm{~b}$, when the acoustic frequency increases, shear stress has an increasing trend until reaching a maximum at $f=5 \mathrm{MHz}$ (resonance frequency). After this point, the increase in the frequency decreases the shear stress. The maximum value of shear stress is when bubble oscillates at resonance frequency and it is about $12 \mathrm{kPa}$. When the bubble oscillates at its resonance frequency, it absorbs and therefore emits more energy; thus, it can create more intense velocity gradient in the transmitting medium, resulting in stronger mechanical stress.

In addition to the acoustic parameters, microbubble radius and microbubble shell have notable effects on bubble dynamic and therefore on vessel wall. In the next two sections, we investigated the effects of these parameters.

\section{The effects of shell viscoelastic} parameters

We herein evaluated the influence of micro-

a
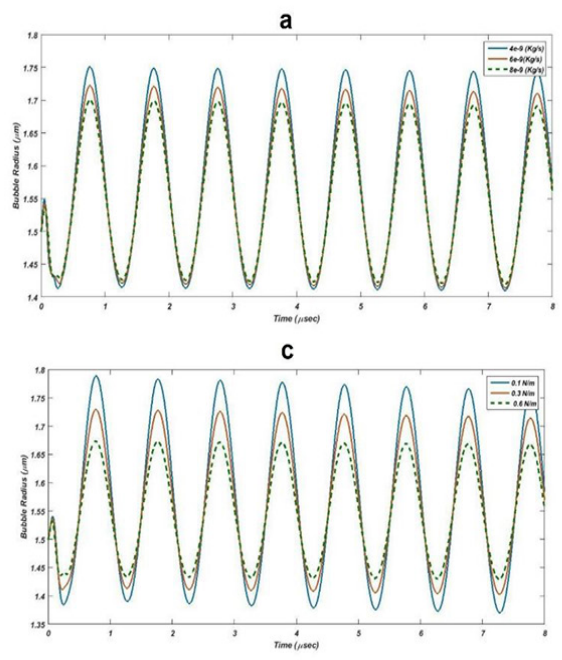

bubble shell viscosity on bubble dynamic and mechanical stress. Microbubble with initial radius of $1.5 \mu \mathrm{m}$ was placed in the center of a vessel with $3 \mu \mathrm{m}$ radius and $5 \mathrm{MPa}$ stiffness. Acoustic pressure amplitude and frequency were set to be $0.25 \mathrm{MPa}$ and $1 \mathrm{MHz}$, respectively. Shell elasticity had a constant value of $0.54 \mathrm{~N} / \mathrm{m}$ and shell viscosity varied between 4 $\times 10^{-9}-9 \times 10^{-9} \mathrm{~kg} / \mathrm{s}$. These shell parameters are in the range of values reported for Definity microbubbles $[27,28]$. Figure 7 a shows the bubble radial oscillation versus time for different shell viscosity. When the shell viscosity is low, the bubble can be activated more easily. By increasing the shell viscosity from $4 \times$ $10^{-9} \mathrm{~kg} / \mathrm{s}$ to $9 \times 10^{-9} \mathrm{~kg} / \mathrm{s}$, the maximum bubble radius decreases from $1.74 \mu \mathrm{m}$ to $1.69 \mu \mathrm{m}$. A more viscose shell has a substantially more damping effect on bubble oscillation leading to lower oscillation amplitudes. Furthermore, by increasing the shell viscosity, the compression velocity of the bubble wall decreases linearly from $1.05 \mathrm{~m} / \mathrm{s}$ to $0.85 \mathrm{~m} / \mathrm{s}$. A decreasing trend was expected for wall stress by increasing shell viscosity, but wall stress has a negligible increasing trend by increasing the shell

b

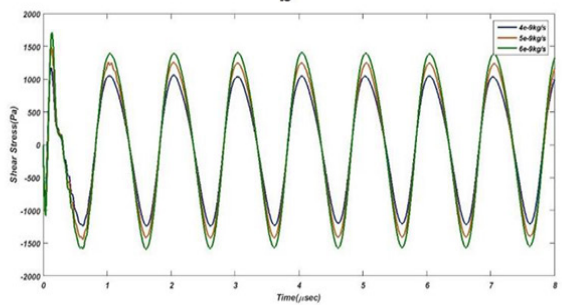

d

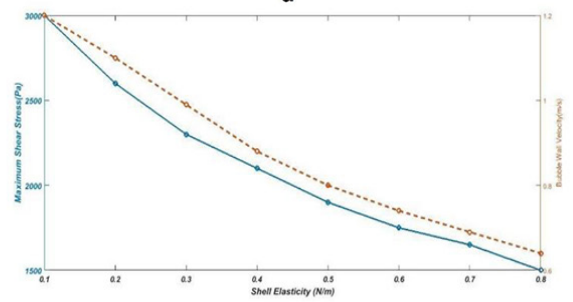

Figure 7: a) Radial oscillation of microbubble versus time for different shell viscosities. $P_{a}=0.25$ $M P a, f=1 \mathrm{MHz}$. b) Oscillating wall shear stress versus time for different shell viscosities. $P_{a}=0.25$ $M P a, f=1 \mathrm{MHz}$. c) Radial oscillation of microbubble versus time for different shell elasticities. $P_{a}=0.25 \mathrm{MPa}, f=1 \mathrm{MHz}$. d) Maximum shear stress and bubble wall velocity versus shell elasticity. 
viscosity as seen in Figure 7b. Beside the shell viscosity another parameter affecting the bubble dynamic is shell elasticity or stiffness. Therefore, we also investigated the effects of shell elasticity. The parameters used here are the same parameters used in the previous section except the shell that had a fixed viscosity of $1 \times 10^{-8} \mathrm{~kg} / \mathrm{s}$ and the varied elasticity of $1-8$ $\mathrm{N} / \mathrm{m}$. Similar to the previous section, as the stiffness of the shell increases, the amplitude of bubble oscillation decreases (Figure 7c) and the maximum bubble wall velocity decreases. As shown in Figure 7d, bubble wall velocity and vessel wall shear stress have the same trend where the increase in the shell stiffness, from $0.1-0.8 \mathrm{~N} / \mathrm{m}$, decreases the bubble wall velocity from $1.2-0.64 \mathrm{~m} / \mathrm{s}$ and the maximum shear stress from 3000-1500 Pa.

\section{The effects of bubble radius}

Figure 8 shows the effect of bubble radius on exerted shear stress on the vessel. For this case, the vessel radius had a fixed value about $5 \mu \mathrm{m}$ and bubbles ranging from 1.5-3 $\mu \mathrm{m}$ in radius was placed in the center of the microvessel. The acoustic pressure had a fixed value about $0.25 \mathrm{MPa}$. As seen in Figure 8, by increasing the microbubble radius, the exerted shear stress also increases. Wall stress is bubble size dependent and also highly depends on distance between microbubble and vessel wall. Figure 8 shows the oscillation of shear stress versus time at the point $r=3$ and $z=3$ on the inner wall of the vessel. It is obvious that the increase in the bubble radius from 1.5-3 $\mu \mathrm{m}$ causes the maximum wall shear stress to increase from 550 to $1400 \mathrm{~Pa}$.

\section{Discussion}

In this study, we developed a 2D axisymmetric model for spherical oscillation of a microbubble inside an elastic microvessel. In our numerical model, the blood was considered as a transmitting medium between the microbubble and vessel wall and there was a two-way coupling between bubble-liquid and liquidvessel wall interfaces. The focus of our study was mainly on the microstreaming induced shear stress on the vessel wall and the effects of different parameters on bubble dynamic and exerted shear stress on the microvessel. This model can help to have a better estimation of bubble dynamic and mechanical stress on the vessel wall to avoid the harmful effects on healthy tissue, thereby increasing the efficiency of the therapeutic procedure.

The current numerical model was validated by R-P motion equation of a bubble inside an unlimited fluid, Figure (1). Not only acoustic parameters, but also the surrounding environ-

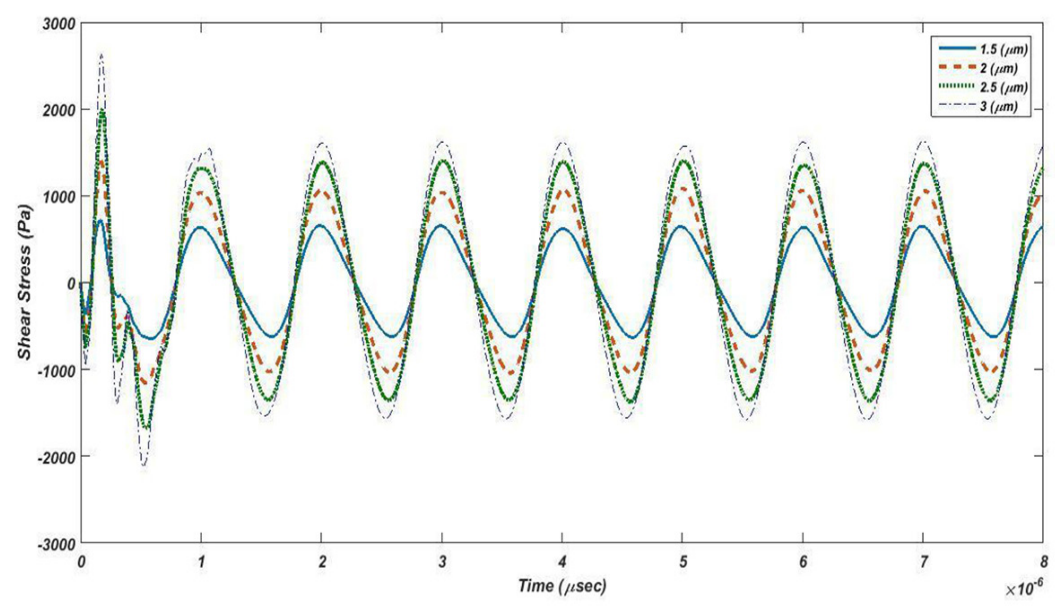

Figure 8: Oscillating shear stress versus time for different bubble radius. $P a=0.25 M P a, f=1 \mathrm{MHz}$, $r_{v}=5 \mu m$ 
ment influences the dynamic behavior of microbubble. The results of our study showed that the presence of vessel wall causes the microbubble experience more damping in radial oscillation and a shift of resonance frequency toward higher frequencies, Figures 2 and 3. These findings are consistent with previous studies $[13,14,29]$. Based on the other studies, an increase in vessel stiffness causes bubble resonance frequency to increase [14, 17].

The stable oscillation of microbubble at low ultrasound field leads to local steady flow, a process named microstreaming. If endothelial cells are present near the oscillating microbubble, microstreaming results in endothelial shear stress. For some applications like blood-brain barrier disruption, local variation in wall shear stress results in straining of the tight junctions connecting the endothelial cells. This strain may cause partial or complete failure of tight junctions and lead to the opening of bloodbrain barrier. As shown in Figure 5, the exerting shear stress on the vessel oscillates with time and location and its maximum value is above the bubble at $z>0$. The value of exerting shear stress on the vessel depends on different parameters. Figure 4 illustrated that by increasing the acoustic pressure, shear stress increases. For the acoustic pressure range used in this study the maximum shear stress at $1 \mathrm{MHz}$ acoustic frequency is below $5 \mathrm{kPa}$. According to Nybrog theory, the microstreaming induced shear stress is proportional to velocity gradient, $G=2 \varepsilon_{0}^{2}\left(\pi^{3} f^{3} \rho\right)^{1 / 2} / R_{0} \eta^{1 / 2}$, where $\mathrm{f}$ is the acoustic frequency and $\varepsilon_{0}$ equals $R-R_{0}$, which is maximum at the bubble resonance frequency [30]. Therefore, in present study when the bubble oscillated at its resonance frequency and at the same acoustic pressure range, the maximum shear stress increased substantially, Figure 6a. Some studies reported that if the shear stress exceed a threshold, finally the endothelial cells would detach [31]. To the best of our knowledge, there is no experimental technique to directly measure the shear stress in a microvessel, but there are some hybrid theoreti- cal/experimental studies, which have calculated the shear stress obtained from microbubble oscillation. Vos et al. reported that when the microbubbles are insonified with $2.25 \mathrm{MHz}$ pulse at $325 \mathrm{kPa}$, the maximum shear stress is about $300 \mathrm{kPa}$ [32]. Dijkink et al. reported that maximum wall shear stress during bubble collapse near a rigid boundary reaches more than $3 \mathrm{kPa}$ [33], which is closer to our results.

For an uncoated microbubble, there are three damping mechanisms reducing the amplitude of oscillation of microbubble as follows: thermal damping, radiation damping and viscous damping (due to the viscosity of surrounding fluid); however, for a coated microbubble, there is fourth source of damping due to viscoelastic coating material. It has been shown that coating is the major source of damping for coated microbubble [34].Van der Meer et al. reported that shell viscosity is responsible for nearly $70 \%$ of total damping and shell elasticity increases the resonance frequency of microbubble by about 50\% [35]. Figure $7 \mathrm{a}$ and $\mathrm{c}$ show that by increasing bubble viscosity and elasticity, radial oscillation of microbubble decreases; although decreasing microbubble radial oscillation does not necessarily lead to lower wall stress. As shown in Figure $7 \mathrm{~b}$ the wall stress increases by increasing shell viscosity, though it is negligible. More studies should be done to shed light on this issue.

Since we have applied some simplifications and approximations in our numerical model, our results are not immune from uncertainties. First, we simulated a single microbubble inside a vessel and ignored bubble-bubble interaction. However, in reality bubbles interact not only with the acoustic field but also with each other also, bubbles have a random position in blood vessel but we considered just one position. In this model, the acoustic pressure was applied directly on the bubble surface; nevertheless, in reality, not only the microbubble but also the vessel and fluid are subjected to the acoustic pressure. Since we used 
Pulsating Microbubble in A Microvessel and its Mechanical Effects

low-pressure amplitudes, ultrasound alone has not had a significant impact on the vessel displacement.

Although our model has some limitations and uncertainties, the results presented in this study are qualitatively consistent with similar studies reported earlier and can give a better insight about how bubble-blood-vessel system are affected by different parameters. By the approach presented in this study, we may have the opportunity to study more complicated physics like the interaction between bubbles and red blood cells in different geometries in future studies.

\section{Conclusion}

In this study, we investigated the effects of different physical parameters on coated micro-bubble oscillation and exerted shear stress on the elastic vessel. Based on our numerical results for different acoustic pressures, when microbubbles are activated at their resonance frequency, which is size-dependent, exerted shear stress on the vessel wall will reach its maximum value. In addition, the results showed that bubbles with higher viscoelastic properties have lower radial oscillation. However, increasing shell viscosity led to higher shear stress. According to our study, softer and larger bubbles are more appropriate for therapeutic application as they can decrease the required frequency and acoustic pressure while inducing the same biological effects.

\section{Acknowledgment}

This study was supported by a grant (No: 32932) from Tehran University of Medical Science.

\section{Conflict of Interest}

The authors declare that there is no conflict of interest regarding the publication of this article

\section{References}

1. Ferrara K, Pollard R, Borden M. Ultrasound microbubble contrast agents: fundamentals and applica- tion to gene and drug delivery. Annu Rev Biomed Eng. 2007;9:415-47. doi: 10.1146/annurev.bioeng.8.061505.095852. PubMed PMID: 17651012.

2. Unger EC, Matsunaga TO, McCreery T, Schumann P, Sweitzer R, Quigley R. Therapeutic applications of microbubbles. Eur J Radiol. 2002;42:160-8. PubMed PMID: 11976013.

3. Kooiman K, Emmer M, Foppen-Harteveld M, van Wamel A, de Jong N. Increasing the endothelial layer permeability through ultrasound-activated microbubbles. IEEE Trans Biomed Eng. 2010;57:29-32. doi: 10.1109/TBME.2009.2030335. PubMed PMID: 19709954.

4. Kooiman K, Foppen-Harteveld M, van der Steen AF, de Jong N. Sonoporation of endothelial cells by vibrating targeted microbubbles. J Control Release. 2011;154:35-41. doi: 10.1016/j.jconrel.2011.04.008. PubMed PMID: 21514333.

5. Sonoda S, Tachibana K, Uchino E, Yamashita T, et al. Inhibition of melanoma by ultrasound-microbubbleaided drug delivery suggests membrane permeabilization. Cancer Biol Ther. 2007;6:1276-83. doi: 10.4161/cbt.6.8.4485. PubMed PMID: 17704642.

6. Endoh M, Koibuchi N, Sato M, Morishita R, Kanzaki T, Murata $Y$, et al. Fetal gene transfer by intrauterine injection with microbubble-enhanced ultrasound. $\mathrm{Mol}$ Ther. 2002;5:501-8. doi: 10.1006/mthe.2002.0577. PubMed PMID: 11991740.

7. Hynynen K, McDannold N, Vykhodtseva N, Jolesz FA. Noninvasive MR imaging-guided focal opening of the blood-brain barrier in rabbits. Radiology. 2001;220:640-6. doi: 10.1148/radiol.2202001804. PubMed PMID: 11526261.

8. Gormley G, Wu J. Observation of acoustic streaming near Albunex@ spheres. The Journal of the Acoustical Society of America. 1998;104:3115-8. doi: 10.1121/1.423903.

9. Wu J. Theoretical study on shear stress generated by microstreaming surrounding contrast agents attached to living cells. Ultrasound Med Biol. 2002;28:1259. doi: 10.1016/s0301-5629(01)00497-5. PubMed PMID: 11879959.

10. Meijering BD, Juffermans LJ, van Wamel A, Henning $\mathrm{RH}$, Zuhorn IS, Emmer $\mathrm{M}$, et al. Ultrasound and microbubble-targeted delivery of macromolecules is regulated by induction of endocytosis and pore formation. Circ Res. 2009;104:679-87. doi: 10.1161/CIRCRESAHA.108.183806. PubMed PMID: 19168443.

11. Ando H, Feril LB, Jr., Kondo T, Tabuchi $Y$, Ogawa $\mathrm{R}$, Zhao QL, et al. An echo-contrast agent, Levovist, lowers the ultrasound intensity required to induce apoptosis of human leukemia cells. Cancer Lett. 2006;242:37-45. doi: 10.1016/j.canlet.2005.10.032. PubMed PMID: 16377079. 
12. Sassaroli E, Hynynen K. Resonance frequency of microbubbles in small blood vessels: a numerical study. Phys Med Biol. 2005;50:5293-305. doi: 10.1088/0031-9155/50/22/006. PubMed PMID: 16264254.

13. Qin S, Ferrara KW. Acoustic response of compliable microvessels containing ultrasound contrast agents. Phys Med Biol. 2006;51:5065-88. doi: 10.1088/00319155/51/20/001. PubMed PMID: 17019026. PubMed PMCID: PMC2847449.

14. Martynov S, Stride E, Saffari N. The natural frequencies of microbubble oscillation in elastic vessels. J Acoust Soc Am. 2009;126:2963-72. doi: 10.1121/1.3243292. PubMed PMID: 20000909.

15. Doinikov AA, Bouakaz A. Theoretical investigation of shear stress generated by a contrast microbubble on the cell membrane as a mechanism for sonoporation. J Acoust Soc Am. 2010;128:11-9. doi: 10.1121/1.3419775. PubMed PMID: 20649196.

16. Wiedemair W, Tukovic Z, Jasak H, Poulikakos D, Kurtcuoglu V. On ultrasound-induced microbubble oscillation in a capillary blood vessel and its implications for the blood-brain barrier. Phys Med Biol. 2012;57:1019-45. doi: 10.1088/00319155/57/4/1019. PubMed PMID: 22298199.

17. Hosseinkhah N, Hynynen K. A three-dimensional model of an ultrasound contrast agent gas bubble and its mechanical effects on microvessels. Phys Med Biol. 2012;57:785-808. doi: 10.1088/00319155/57/3/785. PubMed PMID: 22252221. PubMed PMCID: PMC3367455.

18. Chen C, Gu Y, Tu J, Guo X, Zhang D. Microbubble oscillating in a microvessel filled with viscous fluid: A finite element modeling study. Ultrasonics. 2016:66:54-64. doi: 10.1016/j.ultras.2015.11.010. PubMed PMID: 26651263.

19. Guo X, Cai C, Xu G, Yang Y, Tu J, et al. Interaction between cavitation microbubble and cell: A simulation of sonoporation using boundary element method (BEM). Ultrason Sonochem. 2017;39:863-71. doi: 10.1016/j.ultsonch.2017.06.016. PubMed PMID: 28733016.

20. Hay TA, Ilinskii YA, Zabolotskaya EA, Hamilton MF. Model for the dynamics of a spherical bubble undergoing small shape oscillations between parallel soft elastic layers. J Acoust Soc Am. 2013;134:1454-62. doi: 10.1121/1.4812864. PubMed PMID: 23927185. PubMed PMCID: PMC3749046.

21. Miao H, Gracewski SM, Dalecki D. Ultrasonic excitation of a bubble inside a deformable tube: implications for ultrasonically induced hemorrhage. J Acoust Soc Am. 2008;124:2374-84. doi: 10.1121/1.2967488. PubMed PMID: 19062875. PubMed PMCID: PMC2677346.

22. Mobadersany N, Sarkar K. The dynamic of contrast agent and surrounding fluid in the vicinity of a wall for sonoporation. ArXiv Preprint ArXiv:180208652. 2018.

23. Burton AC. Role of geometry, of size and shape, in the microcirculation. Fed Proc. 1966;25:1753-60. PubMed PMID: 5951414.

24. De Jong N, Cornet R, Lancée Cd. Higher harmonics of vibrating gas-filled microspheres. Part one: simulations. Ultrasonics. 1994;32:447-53. doi: 10.1016/0041-624x(94)90064-7.

25. Hasegawa $H$, Kanai $H$, Chubachi N, Koiwa Y. Noninvasive evaluation of Poisson's ratio of arterial wall using ultrasound. Electronics letters. 1997;33:340-2. doi: 10.1049/el:19970219.

26. Duck FA. Physical properties of tissues: a comprehensive reference book. United Kingdom: IPEM; 2013.

27. Tu J, Swalwell JE, Giraud D, Cui W, Chen W, Matula TJ. Microbubble sizing and shell characterization using flow cytometry. IEEE Trans Ultrason Ferroelectr Freq Control. 2011;58:955-63. doi: 10.1109/ TUFFC.2011.1896. PubMed PMID: 21622051. PubMed PMCID: PMC4495763.

28. Helfield BL, Goertz DE. Nonlinear resonance behavior and linear shell estimates for Definity ${ }^{\mathrm{TM}}$ and MicroMarker ${ }^{\mathrm{TM}}$ assessed with acoustic microbubble spectroscopy. The Journal of the Acoustical Society of America. 2013;133:1158-68. doi: 10.1121/1.4774379.

29. Hosseinkhah N, Chen H, Matula TJ, Burns PN, Hynynen K. Mechanisms of microbubble-vessel interactions and induced stresses: a numerical study. J Acoust Soc Am. 2013;134:1875-85. doi: 10.1121/1.4817843. PubMed PMID: 23967921. PubMed PMCID: PMC3765296.

30. Nyborg WL. Acoustic streaming near a boundary. The Journal of the Acoustical Society of America. 1958;30:329-39. doi: 10.1121/1.1909587.

31. Miller MW. Cell size relations for sonolysis. Ultrasound Med Biol. 2004;30:1263-7. doi: 10.1016/j.ultrasmedbio.2004.07.005. PubMed PMID: 15582225.

32. Vos HJ, Dollet B, Versluis M, De Jong N. Nonspherical shape oscillations of coated microbubbles in contact with a wall. Ultrasound Med Biol. 2011;37:935-48. doi: 10.1016/j.ultrasmedbio.2011.02.013. PubMed PMID: 21601137.

33. Dijkink R, Ohl C-D. Measurement of cavitation induced wall shear stress. Applied Physics Letters. 2008;93:254107. doi: 10.1063/1.3046735.

34. Faez T, Emmer M, Kooiman K, Versluis M, Van Der Steen A, De Jong N. 20 years of ultrasound contrast agent modeling. IEEE Trans Ultrason Ferroelectr Freq Control. 2013;60:7-20. doi: 10.1109/ TUFFC.2013.2533. PubMed PMID: 23287909.

35. Van Der Meer SM, Dollet B, Voormolen MM, Chin CT, Bouakaz A, De Jong N, et al. Microbubble spectroscopy of ultrasound contrast agents. J Acoust SoC Am. 2007;121:648-56. PubMed PMID: 17297818. 